\title{
A Conceptual Framework for Lithium-ion Battery RUL Prediction Using Deep Learning
}

\author{
Shiqiang Zhao ${ }^{1, \text { a }}$ \\ ${ }^{1}$ School of Automation Science and Electrical Engineering, Beihang UniversityBeijing, China \\ a523618033@qq.com
}

\begin{abstract}
Keywords: Lithium-ion Battery, RUL prediction, Deep learning, Deep neural network, Autoencoder. Abstract. In this paper, a conceptual framework for Remaining Useful Life (RUL) prediction of lithium-ion battery integrating deep learning is presented. The main processing stages, i.e., feature extraction, redundant information removal, data preprocessing, DNN model training, RUL prediction and evaluation, are discussed. Finally, a feature extraction method is presented by analyzing a lithium-ion battery data set from NASA AMES Center.
\end{abstract}

\section{Introduction}

With rapid development of modern industry, lithium-ion battery technology has been widely used in vehicle, household equipment, communications, aviation, spacecraft and other fields. Compared with traditional ones, lithium-ion battery has many advantages including high output voltage, high energy density, low self-discharge, long cycle life, high reliability and etc [1-2] . These advantages have brought more widely industrial applications on lithium-ion battery. As the key power supplies of various industrial systems, defections of lithium-ion batteries would typically lead to fatal failures in large numbers of complex systems [3-4]. For example, the National Aeronautics and Space Administration (NASA) once launched the Mars probe. However, as a result of ignorance of battery's status, they rotated solar panels to the direction towards the sun. The battery was over-charged in over temperature, and couldn't recharge. Finally, the lack of power supply led to the loss of the detector [5].

Traditional predicting methods for lithium-ion battery Remaining Useful Life (RUL) are usually divided into two categories: model- driven and data-driven approach [6,7]. The main advantage of the model-based approach is better performance in RUL estimation. However, this kind of approach requires more precise description of the electrochemical degradation process using mathematical or physical or other models. It is difficult to obtain a model with high flexibility and accuracy. In addition, parameters tuning is another obstacle for some model based method. Therefore, it is hard to achieve the expected effect with model based method.

Compared with the model-driven method, the data-driven method mainly depends on "raw" data without requirement of detecting more details, which is similar to the "black box" principle. With the development of computer technology, this method has been improved rapidly and plays a $\mathrm{n}$ increasingly important role in the field of lithium-ion battery RUL prediction. The conceptual framework in this paper employs the principle of data-driven method.

The rest of the paper is organized as follows. Section 2 investigates the related research work concerning autoencoder and deep neural network. The conceptual framework process including feature extracting, redundant information reduction, data preprocessing and model training is discussed in Section3. Section 4 analyzes the features of raw data of lithium-ion battery and presents an approach to feature extracting. Section 5 summarizes the work.

\section{Related Work}

Autoencoder. The autoencoder neural network is an unsupervised learning algorithm that attempts to learn a constant function where the output vector $\mathrm{y}$ is similar to the input vector $\mathrm{x}$. In this case, as the output is similar to the input, the hidden neurons can be used to express the input[8].

Deep Neural Network (DNN). Deep neural network is based on basic artificial neural network model which consists of an input layer, multiple hidden layers and an output layer. Multiple hidden 
layers can provide excellent feature learning ability. Learned features can provide a fundamental characterized profile for the data, and the proper number of hidden units can result in higher prediction accuracy[9]. In the conceptual framework presented in the paper, all layers are fully connected.

\section{Process of Deep Learning Conceptual Framework for RUL Prediction}

The whole process of the deep learning conceptual framework for lithium-ion battery RUL prediction consists of extracting features from original lithium-ion battery data, removing redundant information, preprocessing the data, training the prediction model, and evaluating the performance by comparing the results with different models.

A. Feature extraction. The source data, e.g., voltage, current, temperature and etc., is collected from the charge-discharge cycles of lithium-ion batteries, and grouped to a set of tuples. Because the number of the tuples measured in each charge-discharge cycle always varies, the original data can not be directly input to a prediction model. The characteristic features of each charge-discharge cycle are required to extract. Typical feature extraction methods comprise voltage estimation based feature computing approach [10], time series feature analysis [11], voltage decrease time interval feature computing [12], and geometric feature extraction method [13].

B. Redundant information removal. Typically, there is a positive correlation between information representation and information dimension. As the number of extracted features increases, the number of the highly relevant correlated features grows. But this often leads to model information redundancy and results in low efficiency of model computing. Hence the feature dimensions are needed to reduce to improve model running efficiency. Typical dimension reduction approaches include Principal Component Analysis (PCA) [14], Independent Component Analysis (ICA) [14], and autoencoder. PCA requires that data is subject to the Gaussian distribution. ICA typically depends on prior knowledge of domain. Due to the uncertainty of data distribution, autoencoder could be taken as a feature dimension reduction alternative.

C. Data preprocessing. In order to eliminate the negative effects caused by different scale of feature values, the range of all extracted eigenvalues is transformed to [0,1] by the minimum-maximum normalization method[15]. The minimum and maximum normalization method conversion formula is as follows:

$$
X^{*}=\frac{x-x_{\min }}{x_{\max }-x_{\min }}
$$

$x_{\max }$ is the maximum value of the sample data and $x_{\min }$ is the minimum value of the sample data.

D. DNN model training. Deep network can improve the computation ability of the training model. As each hidden layer can be a layer of the output of non-linear transformation, the deep neural network has more more rich expressive capability than a shallow one. In a deep network, each hidden layer as well as output layer should use non-linear activation function. The trained DNN model is used for RUL prediction of lithium-ion battery. DNN needs to randomly initialize the weight of the depth network before using gradient descent method.

E. RUL prediction and evaluation. By using the training model, the lithium-ion batteries RUL could be predicted. To evaluate the performance of the deep neural network model, the prediction accuracy needs to compare with other approaches such as Bayesian Regression [16], the support vector machine (SVM) [17], Linear Regression [18] and etc. To represent the prediction accuracy, statistics based evaluation methods, e.g., standard deviation, mean squared error, root mean square error (RMSE), could be adopted to evaluate and compare the performance of different prediction models.

\section{Lithium-ion Battery Data Analysis and Feature Extraction Method}

The data source of lithium-ion battery data set is from NASA AMES Center.[25,26]. The battery data set is from the NASA PCoE. 
In this paper, we use the three lithium battery data of B5, B6 and B7 in this data set to accelerate in three different operation modes of charging, discharging and electrochemical impedance measurement under 25 degrees Celsius Degradation of the experiment, and record the observed data. The specific steps are as follows:

(1) Charging process: First, a constant current (Constant Current, CC) 1.5A current lithium battery charge, the voltage was raised to $4.2 \mathrm{~V}$, and then keep the voltage across the battery is a constant voltage Constant Volatage, , CV) at $4.2 \mathrm{~V}$ until the charge current drops to $20 \mathrm{~mA}$.

(2) Discharge process: Firstly, let the battery discharge at a constant current of $2 \mathrm{~A}$ until the voltage of three lithium batteries B5, B6 and B7 drops to $2.7 \mathrm{~V}, 2.5 \mathrm{~V}$ and $2.2 \mathrm{~V}$ respectively.

Experiments need to constantly repeat the battery above three operations to speed up the battery recession process. Impedance measurements provide us with insight into how the battery's internal parameters change as the battery decays. When battery capacity decays to $70 \%$, battery life ends and the experiment ends.

The charging experiment data include: Battery terminal voltage, Battery output current, Battery temperature, Current measured at charger, Voltage measured at charger, Time vector for the cycle.

In a charging process, the changing data involving battery terminal voltage, output current, temperature, voltage measured, current measured with time are shown in Figure 2-6.

The discharging experiment data include: Battery terminal voltage, Battery output current, Battery temperature, Current measured at load, Voltage measured at load, Time vector for the cycle, Battery capacity (Ahr)

In a discharging process, the changing data involving battery terminal voltage, output current, temperature, voltage measured, current measured with time relationship are shown in Fig. 7-11.

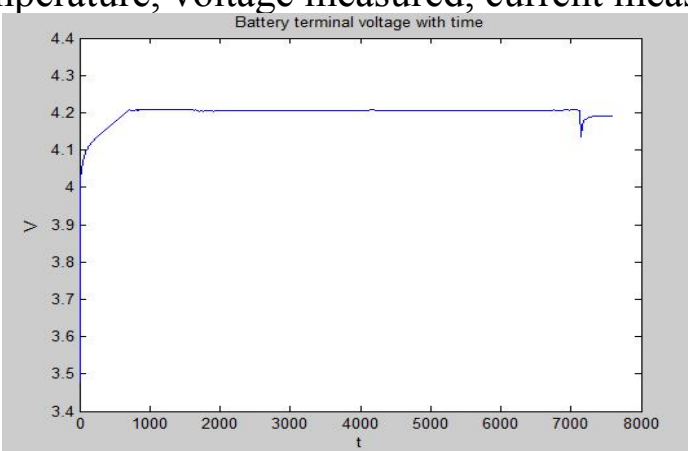

Figure 2. Battery terminal voltage with time

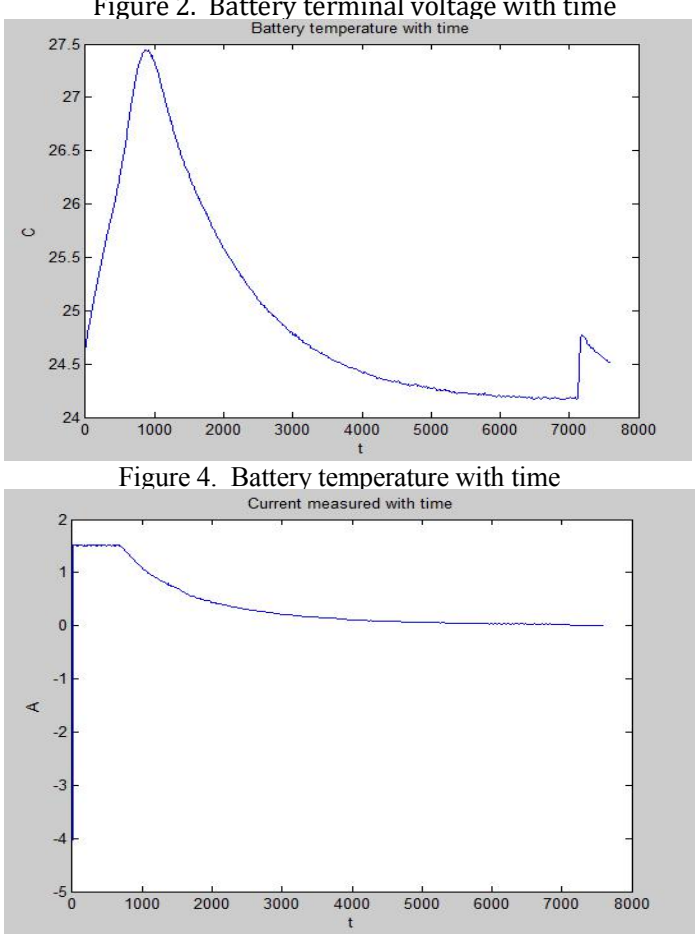

Figure 6. Current measured with time

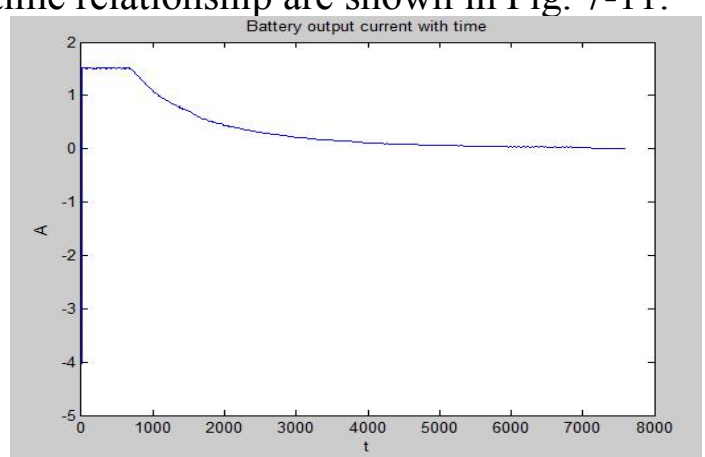

Figure 3. Battery output current with time

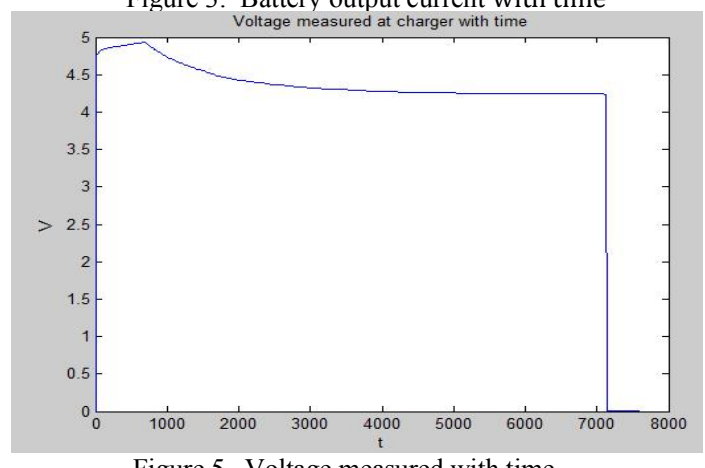

Figure 5. Voltage measured with time

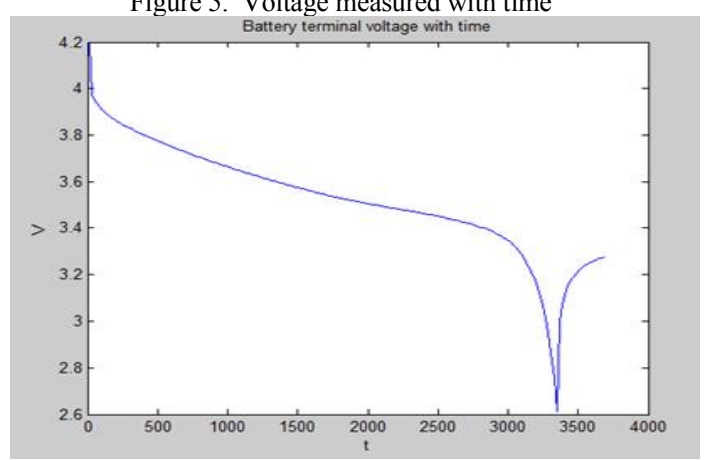

Figure 7. Battery terminal voltage with time 


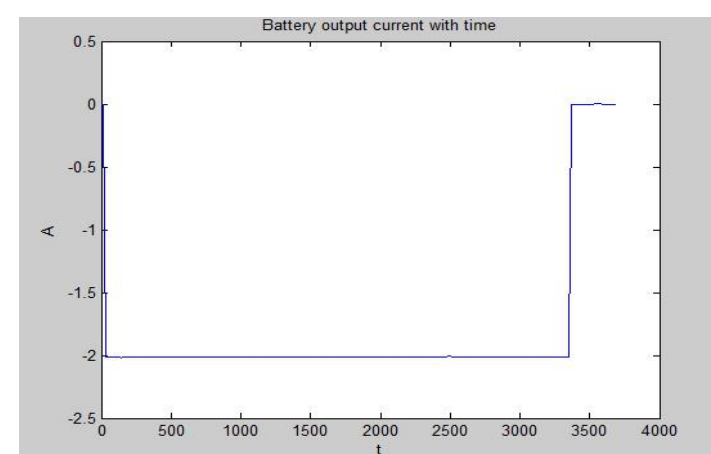

Figure 8 . Battery output current with time

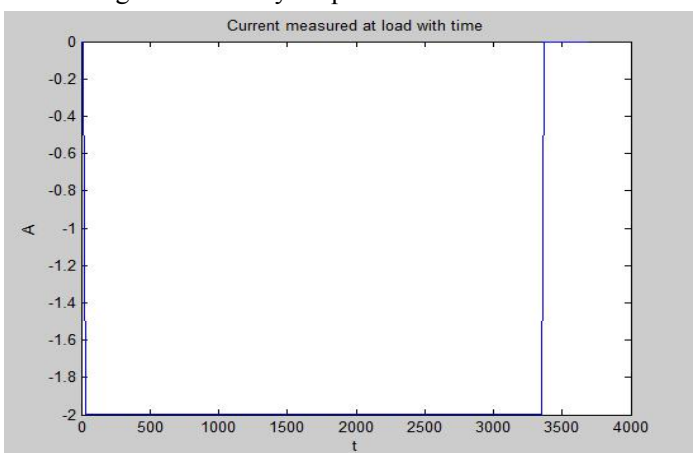

Figure 10. Current measured at load with time

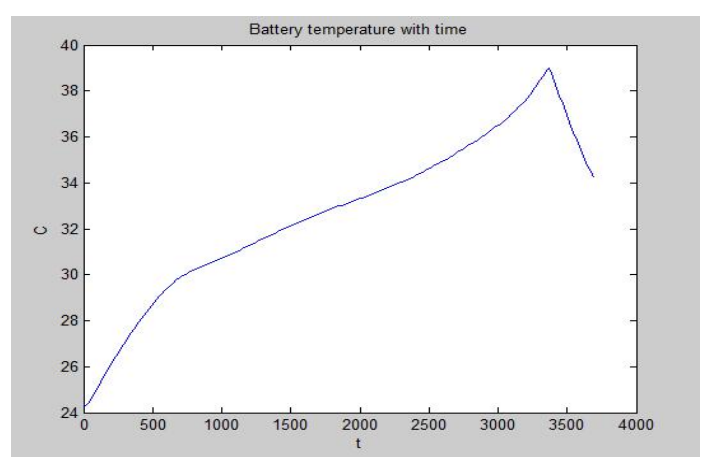

Figure 9. Battery temperature with time

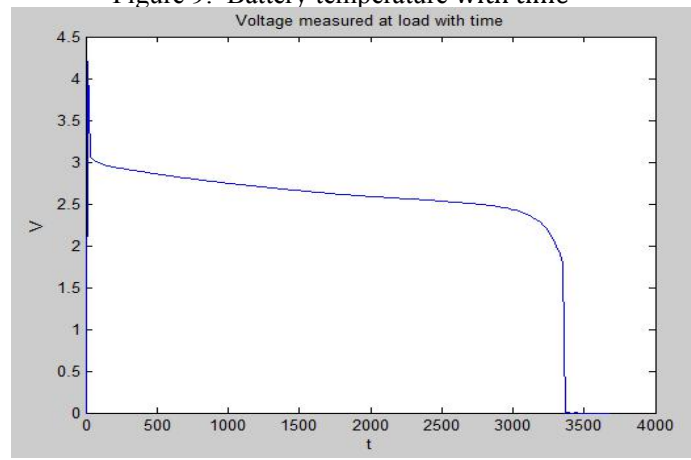

Figure 11. Voltage measured at load with time

We take a geometric feature extraction method to represent and extract the features of lithium-ion battery cycling life. The features extracted from the charging data set as well as discharging data set are shown in Table 1 and Table 2.

Table 1. Features Extracted From Charge Data Set

\begin{tabular}{|l|l|}
\hline \multicolumn{1}{|c|}{ A charge data set } & \multicolumn{1}{c|}{ The feature extracted from the data set } \\
\hline Battery terminal voltage & The maximum and its corresponding time \\
\hline Battery output current & $\begin{array}{l}\text { The time to begin descending and its } \\
\text { corresponding current value }\end{array}$ \\
\hline Battery temperature & The maximum and its corresponding time \\
\hline Current measured at charger & $\begin{array}{l}\text { The time to begin descending and its } \\
\text { corresponding current value }\end{array}$ \\
\hline Voltage measured at charger & The maximum and its corresponding time \\
\hline
\end{tabular}

Table 2. Features Extracted From Discharge Data Set

\begin{tabular}{|l|l|}
\hline \multicolumn{1}{|c|}{ A discharge data set } & \multicolumn{1}{c|}{ The feature extracted from the data set } \\
\hline Battery terminal voltage & The minimum and its corresponding time \\
\hline Battery output current & $\begin{array}{l}\text { The current value at the time of stopping and the } \\
\text { corresponding time }\end{array}$ \\
\hline Battery temperature & The maximum and its corresponding time \\
\hline Current measured at load & $\begin{array}{l}\text { The current value at the time of stopping and the } \\
\text { corresponding time }\end{array}$ \\
\hline Battery capacity & The capacity value \\
\hline
\end{tabular}

\section{Summary}

RUL prediction has great significance on reliability and stability of industrial system that based on lithium-ion power supply. Artificial intelligence and deep learning provide new method for research progress. The experiment presents a deep learning conceptual framework for lithium-ion battery RUL prediction. The main processing stages, i.e., feature extraction, redundant information removal, data preprocessing, DNN model training, RUL prediction and evaluation, are discussed. In addition, a lithium-ion battery data set from NASA AMES Center is analyzed and a feature extraction method is presented. 
The future work is to improve the lithium-ion battery RUL prediction model and finish the experiment by the proposed feature extraction method.

\section{References}

[1] Zhang J, Lee J. A review on prognostics and health monitoring of Li-ion battery[J]. Journal of Power Sources, 2011, 196(15):6007-6014.

[2] He W, Williard N, Osterman M, et al. Prognostics of lithium-ion batteries based on Dempster-Shafer theory and the Bayesian Monte Carlo method[J]. Journal of Power Sources, 2011, 196(23):10314-10321.

[3] Xing Y, Ma E W M, Tsui K L, et al. Battery Management Systems in Electric and Hybrid Vehicles[J]. Energies, 2011, 4(12):págs. 97-106.

[4] Williard N, He W, Hendricks C, et al. Lessons Learned from the 787 Dreamliner Issue on Lithium-Ion Battery Reliability[J]. Energies, 2013, 6(9):4682-4695.

[5] Le D, Tang X. Lithium-ion Battery State of Health Estimation Using Ah-V Characterization[J]. E nvironmental Science \& Technology, 1989.

[6] Peng Y, Liu D, Peng X. A review: Prognostics and health management[J]. Journal of Electronic Measurement \& Instrument, 2010:1007-1015.

[7] Barré A, Deguilhem B, Grolleau S, et al. A review on lithium-ion battery ageing mechanisms and estimations for automotive applications[J]. Journal of Power Sources, 2013, 241(11):680-689.

[8] Lu C, Tao L, Fan H. Li-ion battery capacity estimation: A geometrical approach[J]. Journal of Power Sources, 2014, 261(261):141-147.

[9] Dong Y, Li D. Deep Neural Networks[M]// Automatic Speech Recognition. Springer London, 2015:57-77.

[10] Lin H T, Liang T J, Chen S M. Estimation of Battery State of Health Using Probabilistic Neural Network[J]. Industrial Informatics IEEE Transactions on, 2013, 9(2):679-685.

[11] Widodo A, Shim M C, Caesarendra W, et al. Intelligent prognostics for battery health monitoring based on sample entropy[J]. Expert Systems with Applications, 2011, 38(9):11763-11769.

[12] Liu D, Wang H, Peng Y, et al. Satellite Lithium-Ion Battery Remaining Cycle Life Prediction with Novel Indirect Health Indicator Extraction[J]. Energies, 2013, 6(8):3654-3668.

[13] Lu C, Tao L, Fan H. Li-ion battery capacity estimation: A geometrical approach[J]. Journal of Power Sources, 2014, 261(261):141-147.

[14] Reza M S, Ma J. ICA and PCA integrated feature extraction for classification[C]// IEEE, International Conference on Signal Processing. IEEE, 2017:1083-1088.

[15] Mislick G K, Nussbaum D A. Data Normalization[M]// Cost Estimation: Methods and Tools. John Wiley \& Sons, Inc, 2015:78-104.

[16] Geppert L N, Ickstadt K, Munteanu A, et al. Random projections for Bayesian regression[J]. Statistics \& Computing, 2017, 27(1):79-101.

[17] Cui D, Curry D. Prediction in Marketing Using the Support Vector Machine[J]. Marketing Science, 2005, 24(4):595-615.

[18] Hocking, R. R. The analysis and selection of variables in linear regression[J]. Biometrics, 1976, 32(1):1-49.

[19] Saha B, Kai G, Christophersen J. Comparison of prognostic algorithms for estimating remaining useful life of batteries[J]. Transactions of the Institute of Measurement \& Control, 2009, 31(3):293-308.

[20] J. Y. Liu, X. H. Chen, Q. Liu, and J. Z. Sun, Battery Data Set,NASA Ames Prognostics Data Repository, NASA Ames,Moffett Field, Calif, USA, 2007 